La naissance et la mise en place de la gendarmerie neuchâteloise : d'un corps civil original à l'institution militaire (1809-1850)

Philippe Hebeisen

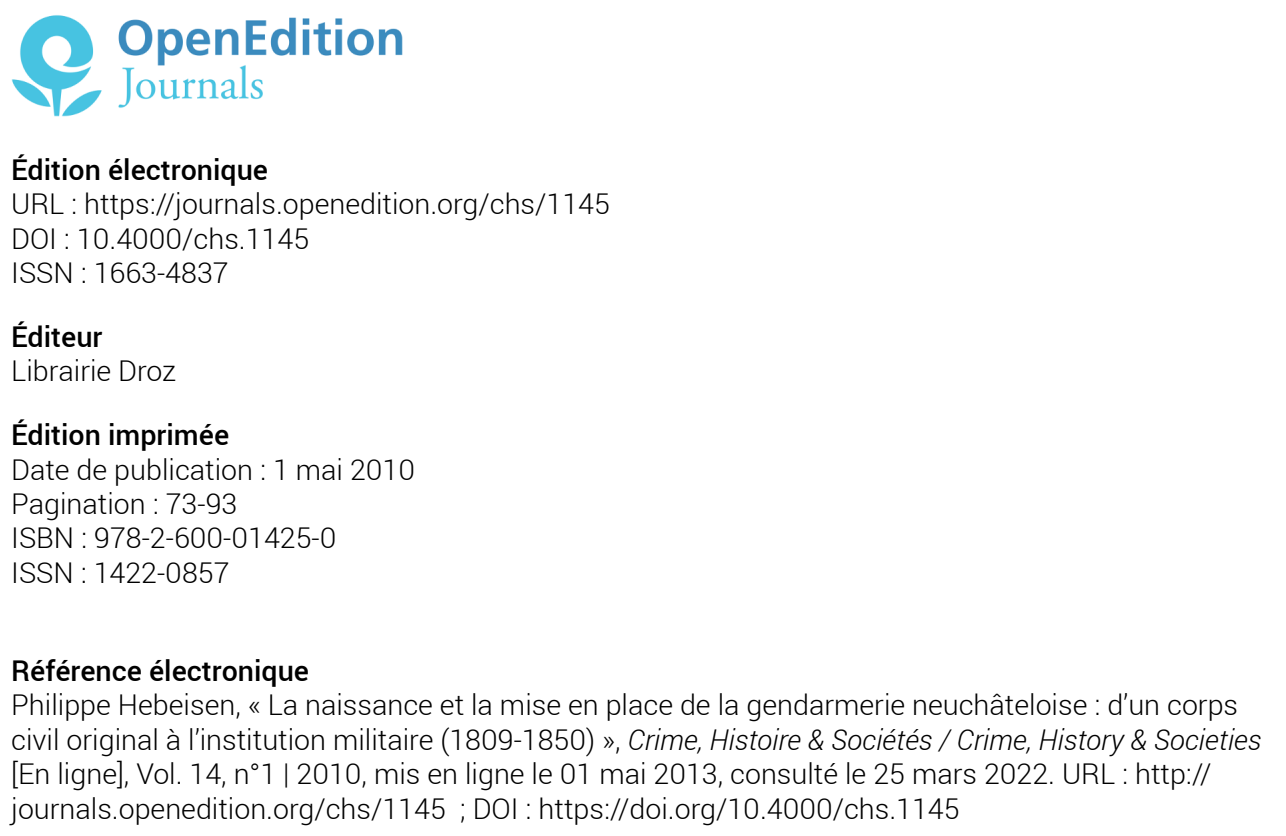




\title{
La naissance et la mise en place de la gendarmerie neuchâteloise d'un corps civil original à l'institution militaire (1809-1850)
}

\author{
Philippe Hebeisen ${ }^{1}$
}

\begin{abstract}
Créée dans le contexte des conquêtes napoléoniennes, la gendarmerie neuchâteloise résiste pourtant à l'acculturation. La maréchaussée dont elle est issue change bien de nom, mais pour l'essentiel demeure, prenant ainsi le contrepied du processus qui a alors lieu en Europe, dans laquelle le modèle français s'impose. Ce n'est que dans une deuxième phase, sous l'Ancien Régime suisse finissant, que la transformation en une véritable gendarmerie est amorcée, au carrefour des influences prussienne, française, mais essentiellement suisses. L'institution n'en demeure pas moins originale, puisqu'elle se distingue de ses consœurs «européennes» par son statut civil. Ce n'est qu'en 1849 qu'elle deviendra un corps militaire de plein droit.
\end{abstract}

The gendarmerie force of Neufchatel, although, established in the context of the Napoleonic conquests of Switzerland, nevertheless resisted adaptation to the French model. Instead, despite changing its name to gendarmerie, it maintained in essence the characteristics of the Maréchaussée force that it had originally been. It thereby went in an opposite direction to the process which took place in other parts of Europe, where the French gendarmerie model was introduced. It was only at a later stage, towards the end of Swiss Ancien Régime, that the development towards a proper gendarmerie force was undertaken, in an amalgamation of Prussian and French influences, but quintessentially Swiss. The Neufchatel gendarmerie remained nevertheless a unique institution, distinguishing itself from European sister-organisations by being a non-military force, that only developed into a military force by 1849.

\footnotetext{
Philippe Hebeisen, doctorant FNS, prépare une thèse de doctorat en cotutelle, sous la direction des profs. Laurent Tissot (Université de Neuchâtel, Suisse) et Jean-Noël Luc (Université Paris IV-Sorbonne), intitulée provisoirement «Le rôle de la police en Suisse occidentale (Neuchâtel, Fribourg, Berne). Institutions, acteurs, pratiques (1848-1914)». Il est également rattaché au Centre de recherches en histoire du XIX ${ }^{\mathrm{e}}$ siècle (Paris I-Paris IV) et travaille dans le cadre du projet de recherche FNS mené par le prof. Nadège Sougy «Le Livre: une marchandise comme une autre? Histoire d'un négoce en zone transfrontalière (1769-1789)». Il a notamment publié «Entre légitimation et reconnaissance: la réintroduction du serment de la gendarmerie neuchâteloise (1840)», in Actes du colloque CUSO: Troisième cycle d'histoire moderne et contemporaine, Universités de Fribourg/Genève/Lausanne/Neuchâtel, 22-23 Novembre et 3-4 Décembre 2007, à paraître 2010; Une histoire de la frontière neuchâteloise durant la Seconde Guerre mondiale: administration et politique face aux réfugiés, Les Verrières (1939-1945), préf. de Marc Perrenoud, conseiller scientifique de la Commission indépendante d'experts Suisse - Seconde Guerre mondiale (1997-2001), Neuchâtel, Alphil, 2007; «La frontière neuchâteloise pendant la Seconde Guerre mondiale: le cas des Verrières (Suisse), 1942-1943», in Robert Belot (dir.), Claude Hauser, Francis Python, Laurent Tissot (coll.), Guerre et frontières : la frontière franco-suisse pendant la Seconde Guerre mondiale, Panazol/ Neuchâtel, Lavauzelle/Ed., Alphil, 2006, pp. 293-310.
} 
Depuis le Pacte fédéral de 1815, Neuchâtel occupe une position singulière au sein de la Confédération suisse, puisque cet ancien pays allié du Corps helvétique $^{2}$ devient à la fois un canton souverain tout en demeurant une principauté prussienne. Ce double statut fait de Neuchâtel un cas unique en Suisse ${ }^{3}$.

Principauté prussienne depuis 1707, le lien entre Neuchâtel et le royaume allemand est fondé sur le régime d'union personnelle. Selon ce dernier, la principauté n'est pas en droit une véritable province prussienne, mais elle est liée personnellement au roi par des rapports politiques et juridiques particuliers, garants de l'indépendance, des institutions et des instruments administratifs et politiques du pays. Pendant toute cette période et jusqu'en 1848, le pays conserve donc ses institutions et sa subdivision en multiples circonscriptions civiles héritées du Moyen Âge. L'élément central du pouvoir est le Conseil d'État ${ }^{4}$, oligarchique et patricien, qui lui aussi perdure à la tête du pays ${ }^{5}$. Le court intermède français ne changera pas fondamentalement la donne ${ }^{6}$. Le maréchal Alexandre Berthier règne sur le territoire de 1806 à 1814, après que celui-ci eut fait l'objet d'un échange entre la Prusse et la France. Notons néanmoins que par rapport au devenir de la Suisse, la situation politique de Neuchâtel est particulière, puisqu'elle n'est pas occupée directement par le Grand Empire.

Dans ce contexte, et malgré des traits communs évidents avec la police suisse, Neuchâtel occupe une place à part, non seulement du point de vue de sa situation politique, mais aussi du point de vue du développement particulier de son corps de gendarmerie. Pour saisir les modalités de cette genèse, il est nécessaire de s'intéresser à la question du statut de la gendarmerie neuchâteloise (police militaire, police civile ou police civile militarisée), ce qui nous amène à nous interroger sur l'origine du ou des modèles policier ou gendarmique qui ont inspiré les autorités neuchâteloises, entre création locale et influences étrangères. Sous des traits a priori exotiques, ce questionnement s'insère en fait très bien dans les discussions relatives à la construction et à la circulation des savoirs policiers en Europe, dans la perspective d'une «histoire européenne des polices $»^{7}$. De plus, l'histoire du corps de gendarmerie neuchâtelois permet, par un jeu de miroir, de mettre en lumière les origines du système policier, c'est-à-dire gendarmique, suisse.

Pour comprendre l'importance du tournant pris entre 1848 et 1850 , après que la révolution de 1848 eût renversé le régime prussien et instauré la république à Neuchâtel, il nous faut revenir quelque peu en arrière afin d'étudier dans quelle mesure et jusqu'à quel point la fin de l'Ancien régime neuchâtelois a constitué le «laboratoire» de police de la gendarmerie républicaine. Le présent papier s'attachera donc

Nom de la Suisse avant 1798, cf. art. «Corps helvétique», DHS (3, p. 594).

V. art. «Confédération » et «Confédération suisse», DHS (3, pp. 458-465 et 468-469), ainsi que «Neuchâtel (canton)», DHS [version électronique], url [http ://www.hls-dhs-dss.ch/textes/f/F7397.php], version du 05.08.2009.

4 Nom du gouvernement de Neuchâtel ou d'un canton suisse.

5 Henry (1984,pp. 48-51). Pour un aperçu plus large du contexte institutionnel neuchâtelois du XVIII ${ }^{\mathrm{e}}$ et du début du XIX ${ }^{\mathrm{e}}$ siècle, cf. Henry (1991, pp. 66-90).

6 Courvoisier $(1959,2002)$; Henry $(2004,2003)$.

7 Pour reprendre les mots de Vincent Milliot (2008, p. 31). 
à décrire les étapes par lesquelles passe l'institution neuchâteloise, afin de mettre en relief, à chaque étape, les influences qui sont à l'œuvre dans ce processus de construction, qui mène d'une gendarmerie de nom vers un corps s'approchant de plus en plus d'une gendarmerie de statut.

\title{
I - LA CRÉATION DE LA GENDARMERIE NEUCHÂTELOISE: ENTRE INFLUENCE FRANÇAISE ET STATU QUO NEUCHÂTELOIS
}

\begin{abstract}
À Neuchâtel, la gendarmerie voit le jour sous le règne du maréchal Berthier, homme-lige de Napoléon, qui le fit prince et duc de Neuchâtel (1806-1814). Par le décret de Berthier du 29 avril $1809^{8}$, une gendarmerie à pied succède dès le $1^{\text {er }}$ juin 1809 à la maréchaussée laborieusement mise en place dans la seconde moitié du $\mathrm{XVIII}^{\mathrm{e}}$ siècle ${ }^{9}$. L'inspiration française se réduit alors à cette option terminologique ${ }^{10}$. La nouvelle gendarmerie reprend en effet à son compte la principale fonction de l'organe qui l'a précédée, soit l'élimination de la mendicité et du vagabondage, tâche pour laquelle elle avait été dotée de pouvoirs prévôtaux, qui sont maintenus ${ }^{11}$. Retenons néanmoins d'emblée que cette création survivra au régime Berthier.

Tout comme le reste de l'administration neuchâteloise a échappé en grande partie au système centralisateur français (pas d'assimilation directe du modèle politicoadministratif de la Grande Nation, dans l'esprit neuchâtelois de résistance à tout modèle imposé de l'extérieur ${ }^{12}$, l'organisation policière de la principauté ne s'est pas faite selon le modèle d'un corps militaire ${ }^{13}$, contrairement à la Gendarmerie impériale - puis nationale - en France. Malgré le nombre de projets élaborés dans ce but, aucun ne voit le jour ${ }^{14}$. Une nouvelle mouture est présentée par le Conseil d'État en décembre 1808. Dépouillée de tout ce qui la rendait coûteuse, elle ne prévoit plus,
\end{abstract}

8 Archives de l'État de Neuchâtel (désormais AEN), série Gendarmerie, AC 522/42, travée (tr.) 755, dossier 6/VII, copie imprimée du décret du 29 avril 1809 et copie d'un arrêt du Conseil d'État du 23.5.1809 concernant l'engagement de neuf nouveaux gendarmes suite au décret de Berthier; décret du prince Berthier du 29 avril 1809 créant la gendarmerie, $R P O$ (1, pp. 185-186).

9 Sur la mise en place et le rôle de la maréchaussée, cf. Henry (1984, pp. 150-156, 200-202 et 507510). Quant à la gendarmerie, on lira avec profit les seules pages écrites à ce propos in Courvoisier (1959, pp. 272-275).

10 Le cas neuchâtelois se distingue du cas espagnol, qui connaît aussi des «gendarmeries de nom» qui ne correspondent pas à une exportation stricte du modèle français, cf. Lepetit (2009). Contrairement au phénomène ibérique, la gendarmerie de la principauté est créée sur demande du gouvernement local, n'est pas utilisée comme force de contre-guérilla (il n'y a pas lieu d'en faire cet usage), ne coexiste pas aux côtés de la gendarmerie française - Lepetit (2006) - à laquelle elle ne sert donc pas de force supplétive.

11 En France, la juridiction prévôtale disparaît en 1790, avant même la fin de la maréchaussée (1791), cf. Brouillet (2003, p. 40).

12 Henry (2003, pp. 278 et 291-292).

13 Le modèle gendarmique français, dans l'acception qui est la nôtre, pourrait être résumé aux caractéristiques suivantes: il s'agit d'un corps au statut militaire et pas seulement organisé militairement, hiérarchisé et subdivisé en échelons hiérarchiques (les grades) et dépendant d'un ministère ou d'un Département militaire, pour ce qui est de la Suisse.

14 Courvoisier (1959, pp. 272-275); AEN, MCE, CP 33/154, tr. 797, p. 1081-1089, 4.12.1806, projet décret du Conseil d'Etat pour l'organisation d'une gendarmerie sur une base militaire. AEN, Fonds Berthier, 4.C-44 a-c, [juin 1808], projet du général français Dutaillis qui s’inspire largement de celui du Conseil d'État. 
pour toute amélioration, qu'une augmentation du corps de neuf gendarmes supplémentaires, avec pour tâche de chasser les vagabonds, prévenir le crime, saisir les malfaiteurs, réprimer la mendicité, surveiller l'exécution des décrets du prince notamment pour ce qui concerne la chasse, l'importation des vins et des liqueurs ${ }^{15}$.

Le projet finalement adopté par Berthier ${ }^{16}$ en avril 1809 se contente d'augmenter le nombre du personnel policier de sept archers de maréchaussée déjà existants à seize gendarmes ${ }^{17}$ et d'imposer l'extension de la juridiction de la gendarmerie à l'ensemble de la principauté. L'absence de toute mention de modification de statut indique que le directeur de la gendarmerie, comme le directeur de la maréchaussée avant lui, demeure un fonctionnaire civil ${ }^{18}$. Le statut de cette nouvelle police suit celui de son chef et les gendarmes sont donc des fonctionnaires civils eux aussi. Neuchâtel se démarque ainsi fondamentalement des autres pays européens occupés par la France, qui tous adoptent le modèle militaire de leur occupant et le conservent souvent au delà de son départ ${ }^{19}$. Autre constat d'importance, il n'y a pas non plus d'intervention d'experts français dans la principauté, comme ce fut le cas, par exemple, dans les départements belges, italiens et rhénans occupés par la France ${ }^{20}$.

Dans le contexte suisse, Neuchâtel se différencie aussi profondément des autres cantons, où la plupart des gendarmeries sont créées en 1803/1804, dans la phase de transition entre la République Helvétique et la Suisse de l'Acte de médiation. Elles se constituent toutes en organisations militaires, sinon à leur début, du moins rapidement $^{21}$. La police de la principauté prussienne se distingue alors non seulement par sa date de création relativement tardive ${ }^{22}$, mais aussi par le statut civil de sa gendarmerie $^{23}$ ! Cependant, elle partage avec ses sœurs suisses le service en uniforme, des tâches similaires (sécurité des chemins et des routes, la chasse aux vagabonds et mendiants, le transport des lettres et directives des autorités), le fait de recevoir ses ordres des chefs de juridiction, la façon de déterminer leurs effectifs en fonction de

15 AEN, Fonds Berthier, 4. D-1 f, rapport du Conseil d'État au prince Berthier, 27.12.1808.

16 Courvoisier 1959, p. 274 n. 1 et supra n. 2.

17 Chiffre très en-deçà des effectifs nécessaires, déjà estimés à 50 hommes en 1709 , cf. Perregaux (1900, p. 105).

18 AEN, Fonds Berthier, I, «Essai sur l'état de la Principauté de Neuchâtel» [1806], pp. 125-130: «Chapitre 8. De la Maréchaussée».

19 Cf. Emsley (1999b); Broers (1999); Luc (2002, pp. 395-472).

20 Cas étudiés par Aurélien Lignereux (travaux présentés notamment à Lille les 6-7 juin et 4-6 décembre 2008, encore inédits). La présence d'experts français est aussi signalée plus tard, en Turquie par exemple, cf. Lévy (2008).

21 Bieri (2004). Il s'agit alors soit de créations ex nihilo, soit de refondations de structures existantes, parfois sans suppression des autres institutions impliquées dans les tâches de maintien de l'ordre et de police.

22 Font aussi exception le canton de Genève, la partie francophone du canton de Berne (ancien évêché de Bâle), intégrés dans des départements français et le Valais, République satellite de la France. Les modalités de création de la gendarmerie et la force du modèle français n'ont pas eu pareille ampleur partout, cf. Porret (2003); Arnet (1954, pp. 59-69), Suratteau (1965, pp. 421-425, 453-456); Bandelier (1980, pp. 140-148); Arlettaz (2003).

23 Concernant le statut social du gendarme, nous nous permettons de renvoyer à notre contribution Entre légitimation et reconnaissance : la réintroduction du serment de la gendarmerie neuchâteloise (1840) (à paraître in Actes du colloque CUSO 2007). Cf. aussi infra. 
l'appréciation de la situation des gouvernements ${ }^{24}$, le financement par un impôt sur les communautés et par les caisses de l'État.

Dans cette première phase, le nombre d'hommes directement au service du gouvernement passe encore de seize gendarmes en 1809 à dix-neuf l'année suivante, contingent qui demeure canonique jusqu'en 1828. Les tâches de la gendarmerie sont alors de surveiller, punir et de lutter contre les vagabonds (particulièrement les vagabonds étrangers), mendiants et rôdeurs; surveiller les forêts et la chasse, réprimer la contrebande des vins et autres abus et assurer la police des routes ${ }^{25}$. Cette institution est bien perçue par la population et ne connaîtra plus de changements notables, sa forme ayant été fixée pour les vingt années à venir.

\section{II - LA RÉORGANISATION DE LA GENDARMERIE (1824-1845)}

Une étape-clé vers une centralisation plus marquée des tâches policières de l'État passe par un processus remaniant la gendarmerie en profondeur, visant une meilleure autonomie du corps par rapport aux chefs des juridictions civiles, dont son action dépend en grande partie, et une amélioration qualitative de cette troupe par un meilleur recrutement ${ }^{26}$. D'une police destinée d'abord essentiellement aux campagnes et à leurs communautés, elle se mue lentement aussi en police des villes (Le Locle, La Chaux-de-Fonds, Neuchâtel), jusqu'à acquérir une véritable stature de police d'État, centralisée, plus efficiente et participant à la sûreté de l'État ${ }^{27}$, notamment par une surveillance policière de la population neuchâteloise et étrangère.

\section{1 - La redéfinition et la clarification de ses tâches (avant 1831)}

La fin de l'année 1824 voit l'entrée en fonction d'Armand-Frédéric de Perregaux $^{28}$ dans la charge de directeur de la gendarmerie, corps dont il sera le grand architecte. Ce même jour est marqué par l'adoption par le Conseil d'État du premier règlement de la gendarmerie. Ce nouveau code définit clairement les tâches de l'institution. Il délimite également son action pour apaiser les craintes du Conseil d'État vis-à-vis des pouvoirs du chef de la police. Ainsi, «les gendarmes sont chargés de la recherche, de l'arrestation et du transport des criminels et des individus signalés; b) de la surveillance habituelle et journalière des gens sans aveu, suspects de vagabondage; de la recherche des individus réclamés et signalés par les États voisins; c) de la suppression de la mendicité, et de la conduite des mendiants et rôdeurs dans leurs

24 Bieri (2004, pp. 137-138). D'autres fonctions, comme la capture des criminels et l'escorte de détenus, dont les gendarmes suisses ont à s'occuper dès le début, ne seront réalisées que plus tardivement par la gendarmerie neuchâteloise, en raison de l'archaïsme du système judiciaire neuchâtelois et du rôle que les huissiers de justice et les justiciers (juges) y jouent encore, cf. infra.

25 Courvoisier (1959, p. 274).

26 Question que nous ne pouvons aborder ici faute de documentation suffisante pour la période concernée. Cf. néanmoins ch. 2.5 infra.

27 Au sens que l'entend Rousseaux (2006, pp. 24-26).

28 A.-F. de Perregaux (1790-1873), notamment directeur de la gendarmerie (1824-1845) et Conseiller d'État (1831-1848), a été l'artisan de l'affirmation de la gendarmerie neuchâteloise (article à paraître dans Revue historique neuchâteloise en 2010). 
communes, s'ils sont du pays, ou hors de l'État s'ils sont étrangers [...]; d) ils sont chargés de plus de surveiller l'exécution des décrets, mandements et ordonnances pour la sanctification du dimanche, pour la police des cabarets, pour la police des chemins et des voituriers, pour la chasse, pour la pêche, pour la police sanitaire du bétail, et généralement de tous les règlements de police qui intéressent la sûreté publique $\gg^{29}$ et de rapporter à l'officier de la juridiction, ou à son représentant, toutes les infractions à ces textes.

Les tâches décrites aux points b) et c) découlent directement de la justice ou procédure expéditive héritée du XVIII ${ }^{\mathrm{e}}$ siècle, cristallisée dans l'article 88 de la Loi de procédure criminelle de 1835 , qui confirme cette justice prévôtale comme attribut de la gendarmerie, en continuité de la maréchaussée, dont elle hérite ces compétences judiciaires ${ }^{30}$. D'ailleurs, pour orienter le gendarme dans l'exercice quotidien de ses tâches, il est nouvellement aidé (dès 1826) par le recueil de règlements ${ }^{31}$ qui lui est remis à son entrée dans le corps et qu'il doit restituer lorsqu'il le quitte. Ce petit livret ne recense pas moins de 37 mandements, arrêts, décrets, ordonnances, extraits d'arrêts et d'ordonnances, règlements soumis à la surveillance et à l'exécution des gendarmes, ce qui témoigne de l'extension de ses tâches depuis 1810 .

En mars 1826, l'on considère néanmoins les attributions de la gendarmerie comme réglées $^{32}$. Les règlements de 1827 et 1840 ne sont que du toilettage. À cette occasion, les gendarmes se voient confirmés dans leur tâche de surveillance des routes (partagée avec les «gens d'office» des communes et les voyers) ${ }^{33}$, ce d'autant plus que leur construction et leur entretien reviennent désormais à l'État, qui mobilise donc une institution qui lui est toute dévouée, la gendarmerie, pour veiller sur son bien. La pratique révèle que c'est une des tâches privilégiée des gendarmes ${ }^{34}$. En 1827 , leurs interventions dans la surveillance des étrangers sont clarifiées à leur tour $^{35}$, notamment en ce qui concerne l'encadrement des populations de travailleurs saisonniers (vendangeurs, faucheurs, batteurs en grange et autres ouvriers travaillant à la terre, aux récoltes et aux chemins). Mais malgré une bonne volonté affichée, la gendarmerie manque de moyens. En 1829, par exemple, elle ne dispose que de six gendarmes pour combattre la contrebande le long des frontières, nombre insuffisant, même pour Neuchâtel ${ }^{36}$.

29 AEN, MCE, CP 33/173, tr. 797, f ${ }^{\circ}$ 988-993, 13.12.1824: «Gendarmerie. Règlement », article 12, et arrêté de nomination; AEN, série Gendarmerie, AC 522/42, tr. 755, dossier 6/VIII, 7.12.1824, brouillon de ce règlement, qui diffère peu.

30 Henry (1984, pp. 150-156) et $R P O$ (3, pp. 35-72), Loi de procédure criminelle, 16.12.1835, article 88 p. 70. Discussion dans $B O C L$ (1832, 3, p. 464; 1834, 5, pp. 135-136), p. 222 (séance du 19 décembre 1834, à laquelle participe de Perregaux), la loi amendée et adoptée figure pp. 223-246. Les pouvoirs prévôtaux de la gendarmerie, après avoir été restreints momentanément en 1824, ont ensuite été étendus par deux fois, en 1828 et 1840, cf. Christ (2009, pp. 1209-1223 et n. 560, 586, 609).

31 Recueil de règlemen[t]s à l'usage de la gendarmerie, Neuchâtel: C.-H. Wolfrath, 1826 [en fait 1828], 123 p.; ex. tirés des pp. 3-13 et 118-120.

32 AEN, CP 34/20, tr. 775, 6.3.1826.

33 Henry, Jelmini (1991, p. 129); RPO (2, pp. 87-94, article 16) et $R P O$ (3, pp. 222-223).

34 AEN, série Gendarmerie, AC 522/42, tr. 755, dossier 7/VI, Tableau comparatif des délits découverts par la gendarmerie 1826-1847, 5.1.1847.

$35 R P O$ (2, pp. 94-101, articles 11 et 13), dispositions confirmées dans le Règlement concernant les étrangers du 23 juillet $1833, R P O$ (2, pp. 381-388, articles 15 et 16).

36 AEN, série Gendarmerie, AC 522/42, tr. 755, dossier 6/VIII, rapport du directeur de la gendarmerie de Perregaux au Conseil d'État, 12.1.1829. 


\section{2 - La création de la police centrale: le modèle suisse}

La police centrale apparaît en 1821 à Neuchâtel. Elle examine toutes les requêtes des étrangers ${ }^{37}$ dont elle conserve les papiers. Cette création, couplée à la loi sur les étrangers de 1827, représente un pas de plus vers une centralisation administrative des compétences de l'État. C'est le maire de Pierre, premier directeur de la gendarmerie (1809-1824), qui est le premier président de la commission de police (1821$1824)^{38}$ et vraisemblablement son créateur. En grand admirateur de la République de Berne, il l'a créée sur son modèle ${ }^{39}$.

Or, depuis le règlement de 1824 , le directeur de la gendarmerie est sous les ordres de cette commission, dont lui et son secrétaire sont membres ${ }^{40}$, soumission qui est tout à fait nouvelle et résulte de problèmes de politique interne à la principauté. Dans ses nouvelles attributions, la commission de police centrale examine par exemple la question de l'augmentation du nombre de gendarmes $(1827)^{41}$, prépare les dossiers concernant la gendarmerie pour le Conseil d'État et gère ce qui relève de ce que l'on appelle aujourd'hui la police administrative (heure de fermeture des cabarets, heure d'ouverture autorisée pour les débits de vin, etc. ${ }^{42}$ ). Même si le rôle du directeur de la gendarmerie au sein de la commission est prépondérant, celle-ci représente encore un moyen de juguler toute tentative d'accroissement soudain et incontrôlé de son pouvoir, préoccupation chère au Conseil d'État.

La mise sur pied de la police centrale, en 1821 devait aller de pair avec l'élargissement du nombre des gendarmes de 19 à 24 et la création d'un commandement militaire (trois caporaux et un sous-lieutenant sous les ordres du directeur de la gendarmerie) afin qu'ils aient «cette tenue et cette discipline qui en impose aux autres $»^{43}$. Tentative sans lendemain, puisque la gendarmerie demeure un corps civil. L'idéal de militarisation n'est pas abandonné pour autant, puisqu'entre 1830 et 1848, l'uniforme porté par les gendarmes était coupé comme celui des milices neuchâteloises ${ }^{44}$.

\section{3 - L'apparition du brigadier: réminiscence française ou influence prussienne?}

Le modèle gendarmique français refait curieusement surface lorsque des postes de sous-officiers, intermédiaires entre le directeur de la gendarmerie et les

37 AEN, CP 34/19, tr. 775, pp. 426 sqq.; Christ (2000, pp. 51-73). On prévoyait encore de lui confier à l'origine la correspondance et «diverses parties de la police», ce qui n'a pas été le cas de prime abord, cf. n. 46.

38 Cf. $A N(1822-1824)$.

39 Montandon (1969, p. 148); AEN, série Police centrale, AC 522/70, tr. 762, dossier 66/I, «Quelques idées sur la commission de police centrale», demi-feuille de papier filigrane du canton de Berne, concernant la première phase de l'organisation d'une commission de police centrale à Neuchâtel, [1822]; ibid., arrêt donné en Commission de police centrale, 26.1.1824. Règlement de la gendarmerie, articles 2 et 4 , cf. n. 28.

AEN, MCE, CP 33/176, p. 837, 19.11.1827.

42 AEN, MCE, CP 33/180, p. 519, 21.6.1831: Ordonnance relative aux gendarmes, rapport de la commission de Police.

43 AEN, série Gendarmerie, AC 522/42, tr. 755, dossier 6/VIII, brouillon de rapport non daté et non signé, [1821].

44 Bachelin (1890). 
gendarmes, sont mis en place dans les années 1826-1827. En 1826, le directeur de la gendarmerie propose l'augmentation du nombre de ses hommes, qu'il aimerait voir se conjuguer avec la création de quatre postes de sous-officiers, qui n'ont encore ni grade, ni nom ${ }^{45}$. Fin 1827 cependant, leur création est effective, sous le nom de brigadier $^{46}$, grade que l'on ne retrouve pourtant nulle part ailleurs en Suisse, que ce soit dans les milices cantonales ou les états-majors fédéraux.

Cet emprunt à la nomenclature française ${ }^{47}$ est d'autant plus étonnant que le paysage gendarmique suisse influence de plus en plus ce qui se fait à Neuchâtel. Ainsi, en 1826, lorsque la gendarmerie envisage d'augmenter le nombre de ses hommes (pour la première fois depuis 1810 !), des renseignements sont pris quant au nombre de gendarmes présents dans les cantons voisins de Berne, Fribourg, Vaud et Genève ${ }^{48}$.

Toutefois, le paradoxe n'est qu'apparent. En effet, la France n'est qu'un modèle indirect, puisque le terme a transité par la Prusse ${ }^{49}$. Il figure sur un rapport de Berlin concernant l'organisation de la gendarmerie prussienne, daté de décembre 1820 , époque à laquelle l'on songeait encore à militariser son homologue neuchâteloise $\mathrm{e}^{50}$. Il est donc fort probable que lors de l'augmentation du nombre de gendarmes fin 1827-début 1828 , la référence à ce rapport ait permis la création des quatre nouveaux postes de sous-officiers ${ }^{51}$. Cet épisode mis à part, l'influence de la Prusse en matière de police n'est pas prégnante. Des similitudes entre la Prusse et Neuchâtel existent néanmoins dans la manière de s'assurer la fidélité des sujets, puisque les troupes militarisées y demeurent le premier rempart contre des désordres sociaux ${ }^{52}$. Comme par exemple en 1847-1848, lorsque, malgré l'affirmation progressive de la gendarmerie en matière de maintien de l'ordre, le régime royaliste, se sentant menacé, mobilise une fois encore les outils qui lui avaient permis de se maintenir en 1831: les gardes locales, les comités de défenses (en charge de la sécurité et du maintien de l'ordre) et le bataillon soldé ${ }^{53}$.

\section{4 - L'organisation territoriale}

L'établissement des quatre brigadiers révèle une autre bizarrerie. Consécutivement, le territoire neuchâtelois est subdivisé en quatre brigades de gendarmerie,

45 AEN, série Gendarmerie, AC 522/42, tr. 755, dossier 6/VIII, rapport du directeur de la gendarmerie de Perregaux au gouverneur de la principauté-canton, 28.11.1826.

46 AEN, série Gendarmerie, AC 522/42, tr. 755, dossier 6/VIII, le maire de Valangin de Chambrier au gouverneur, 5.11.1827.

47 En France, le grade de brigadier est peu élevé et équivaut à peu près à celui de caporal d'infanterie et a cours aussi bien dans la maréchaussée que dans la gendarmerie, cf. Luc (2005, p. 827).

48 AEN, série Gendarmerie, AC 522/42, tr. 755, dossier 6/VIII, rapport du directeur de la gendarmerie de Perregaux au gouverneur de la principauté-canton, 28.11.1826.

49 Rappelons ici que le Conseil d'État de la principauté-canton devait rendre des comptes réguliers à Berlin, particulièrement sous la Régénération, cf. Gugger (1999).

50 AEN, série Gendarmerie, AC 522/42, travée (tr.) 755, dossier 6/VIII, rapport de Berlin intitulé «Etat für die gesammte Gendarmerie», 30.12.1820.

51 AEN, MCE, CP 33/176, 17.12.1827, p. 937 et 24.12.1827, p. 955 et MCE, CP 33/177, 12.5.1828 et n. 39 .

52 Lüdtke (1982, pp. 143-282); Emsley (1999a, pp. 37, 39).

53 Courvoisier (1982). 
dont les chefs sont installés à Neuchâtel, au Locle, à La Chaux-de-Fonds et au Valde-Travers ${ }^{54}$. Ce fractionnement laisse à penser qu'il s'est fait selon l'ancienne division militaire du pays - qui repose sur les quatre régions naturelles du canton - partagé en quatre «arrondissements de territoire» avant 1814: départements du Val-de-Ruz, du Vignoble, des Montagnes et du Val-de-Travers ${ }^{55}$. Hypothèse quant au partage gendarmique de l'espace qui fonctionne si l'on considère que La Chauxde-Fonds est le poste central pour la «brigade ${ }^{56}$ du Val-de-Ruz, bien que la ville se situe géographiquement dans les Montagnes neuchâteloises.

Or, le règlement militaire de 1823 , antérieur à la création des brigadiers, découpe la principauté en six départements militaires ${ }^{57}$, alors que le paysage gendarmique ne compte que quatre brigades. Il y a donc inadéquation entre ces deux systèmes de division territoriale, découpage sans lien non plus avec les nombreuses juridictions civiles et criminelles qui morcellent le canton. Est-ce à dire que l'on ne peut pas interpréter l'établissement de brigadiers et de «brigades» comme un pas vers la militarisation de la gendarmerie? Non, car cette dissymétrie est plutôt révélatrice des tensions internes au régime et des mutations en cours. Dans ce sens, l'organisation territoriale de la gendarmerie se calque peut-être à dessein sur l'ancien maillage militaire du pays, antérieur à 1815, afin de se démarquer de l'organisation militaire issue du modèle confédéral. Neuchâtel navigue en effet entre aspirations suisses et prussiennes, particulièrement pendant la Régénération ${ }^{58}$, et cette façon de faire montrerait le parti pris des autorités neuchâteloises, tenantes de l'ancien système.

L'indécision quant à la forme à donner à cette gendarmerie, toujours civile, est patente. On hésite, essaie timidement de la doter d'une organisation de type militaire ou paramilitaire. Cela, en flagrante opposition, pourtant, avec le statut des gendarmes, qui ne sont pas soumis à la discipline militaire ${ }^{59}$, dont le grade ne correspond pas à ce qui a cours dans la milice neuchâteloise et qui, de surcroît, sont exemptés du service militaire en leur qualité de «fonctionnaires publics $»^{60}$.

\section{5 - La fusion de la gendarmerie avec la commission de police centrale et sa réorganisation (dès 1832)}

La coexistence de la commission de police centrale et de la gendarmerie implique un pouvoir policier dilué, ce dont le directeur de la gendarmerie de Perregaux est conscient. C'est à la faveur de la Révolution manquée de 1831 et du remaniement en profondeur de l'administration de la principauté qui s'ensuit qu'un nouveau cap dans l'organisation de la gendarmerie neuchâteloise est franchi. La situation est alors très favorable au directeur de la gendarmerie. Son accession au

54 AEN, MCE, CP 33/184, pp. 150-151, 28.1.1833.

55 AEN, Fonds Berthier, I, «Essai sur l'état de la Principauté de Neuchâtel» [1806], pp. 135-145: «Chapitre 10. Des milices »; AEN, MCE, CP 33/162, p. 590, 30.6.1814.

56 Le terme est placé entre guillemets car il n'est jamais employé dans les sources.

57 RPO (1, Règlement militaire du 27 juin 1823, pp. 397-456, spéc. pp. 405-406); Bauer (1948, p. 34).

58 Gugger (2005).

59 RPO (1, Loi sur la discipline militaire du 14 novembre 1820, pp. 359-374). Cette loi s'adresse essentiellement aux milices de l'État. Elle a été confirmée par la Déclaration royale du 24 avril 1826, cf. $R P O$ (2, pp. 69-71).

${ }^{60} \operatorname{RPO}(1$, Règlement militaire du 27 juin 1823, pp. 400, 416, 417, 420, 422, 423). 
Conseil d'État, le 3 novembre $1831^{61}$, offre enfin à cet «homme nouveau ${ }^{62} »$ voulu par le commissaire royal de Pfuel (envoyé depuis Berlin pour soumettre les révolutionnaires) l'occasion et les moyens de réaliser ses desseins concernant la gendarmerie $^{63}$.

Tirant les leçons de l'insurrection, Perregaux dresse un «rapport sur les améliorations dont la police de l'État est susceptible ${ }^{64}$. Revenant sur les rôles de la direction de la gendarmerie et sur celui de la commission ou bureau de police centrale, il propose de les réunir en une seule et même administration, sous le nom de direction de la police centrale, regroupant à la fois la gendarmerie et l'administration qui contrôle les papiers des étrangers. L'exemple de la Suisse sert à nouveau de référence, un organisme semblable existant notamment à Berne ${ }^{65}$. Ce regroupement supprime dans le même temps la tutelle qu'exerçait la commission sur la direction de la gendarmerie, qui retrouve son autonomie. La «nouvelle police» est consolidée par les événements de 1831, à plus d'un titre. C'est elle, et non plus le Conseil d'État, qui centralise dès lors les informations envoyées par les chefs de juridiction

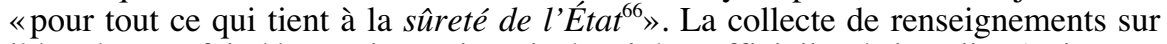
l'état du pays fait désormais aussi partie des tâches officielles de la police (qui y procédait auparavant sans y être obligée). C'est une véritable révolution, car ce genre de correspondance était autrefois reproché à l'ancien directeur de la gendarmerie de Pierre et avait mené à la mise sous tutelle de l'institution par la commission de police, avec laquelle la gendarmerie fusionne début $1832^{67}$.

L'ensemble des mesures prises concourt ainsi, dans le droit, à renforcer notablement les pouvoirs du directeur de la gendarmerie et son rôle en tant que véritable chef de la police, en ne le cantonnant plus simplement à un rôle d'administrateur du corps et de comptable «amélioré». En réalité, de Perregaux avait pris un peu d'avance sur la sanction légale de ces dispositions. Une mainmise plus marquée du gouvernement sur la gendarmerie, dont elle devient véritablement l'instrument, est l'autre résultante des changements entrepris. Le corps de gendarmerie sort ainsi notablement renforcé de la crise.

Dans la foulée, les différents règlements «dont l'observation est remise à la surveillance de la gendarmerie» sont revus en 1832 et restreints par rapport à 1824 . Comme à tous les fonctionnaires publics, il incombe aux gendarmes de veiller à l'exécution des règlements et ordonnances de la principauté. Cependant, ils sont

61 Sur les événements de 1831, cf. Barrelet (1993, pp. 24-28); Scheurer et al.(1987,pp. 161-168); RPO (2, pp. 278-282) et idem, déclaration royale sur l'organisation du Conseil d'État du 31 mai 1832, pp. 294-296; Gugger (1997, pp. 12-17).

62 L'expression est de Henry (1999a, p. 318).

${ }_{63}$ Montandon (1969, pp. 269, 270, 302) et Courvoisier (1982).

${ }_{64}$ AEN, série Police centrale, AC 522/70, tr. 762, dossier 66/I, rapport du directeur de la gendarmerie et Conseiller d'État de Perregaux au commissaire royal de Pfuel, 12.11.1831; v. aussi MCE, CP 33/181, tr. 797, p. 1307, arrêt du Conseil d'État du 13.12.1831.

65 Il s'agit de la Centralpolizeidirektion, dont le chef, à Berne, n'est cependant pas le même que celui de la gendarmerie, cf. $B S$, p. ex. 1846 et Arnet (1954).

66 Nous soulignons. AEN, série Police centrale, AC 522/70, tr. 762, dossier 66/I, rapport du directeur de la gendarmerie au commissaire royal, 12.11.1831.

${ }^{67}$ AEN, MCE, CP 34/21, tr. 775, p. 862, 8.3.1832. 
spécialement chargés de veiller à «la surveillance des gens sans aveu, la suppression de la mendicité, la police des routes, l'escorte des détenus, la garde des prisons, la police des jeux prohibés aux foires, etc., la police des conducteurs d'animaux, saltimbanques, etc., la police de la vente des branches de sapin, la surveillance des auberges et cabarets, la police des chiens ${ }^{68}{ } \gg$. Ce qui est frappant dans les attributions revues en 1832, c'est la disparition des tâches de police judiciaire (recherche et arrestation des criminels et des individus signalés); seul survit le transport des détenus. La fonction qui semblait être la plus nouvelle, à certains égards la plus proche d'une gendarmerie d'État, est abandonnée pour l'instant et redevient l'apanage des anciens fonctionnaires qui en avaient la charge (huissiers de justice des juridictions civiles et criminelles, parfois aidés des justiciers) ${ }^{69}$. C'est ici un point d'achoppement, une façon de faire de l'Ancien régime neuchâtelois que la gendarmerie ne parvient pas à bouleverser. Ceci s'explique par la volonté de préserver les anciennes structures, à la fois garantes et reflet de l'ordre social de la principauté, certes, mais aussi par l'absence de nécessité de changement, puisque le directeur de la gendarmerie lui-même estimait que «les sautiers sont en général suffisants pour mettre à exécution les décrets de prise de corps $»^{70}$.

Parallèlement, on réduit le nombre des gendarmes tout en augmentant leur traitement et en «réformant»le corps par de nouveaux engagements ${ }^{71}$. Ces mesures n'entraînent pas une diminution du pouvoir de la gendarmerie, demande refusée une année plus tôt $\mathrm{t}^{72}$. Ce que l'on vise, ce sont des attributions plus claires et des tâches aux contours beaucoup plus précis, vraisemblablement dans l'optique d'une plus grande participation de la gendarmerie à la répression du mouvement insurrectionnel et à la surveillance de la population. C'est en tout cas ce que tendraient à relever les chiffres de la pratique ${ }^{73}$. La collaboration de la gendarmerie avec les comités de défense royalistes (volontaires armés par le gouvernement et chargés de la sécurité publique en période de troubles), bien attestée, même si elle ne va pas toujours sans $\mathrm{mal}^{74}$, illustre bien le glissement du rôle de la gendarmerie, qui évolue petit à petit vers une véritable police d'État et des tâches de maintien de l'ordre, du moins par le biais de la surveillance de la population. Ce phénomène est évidemment particulièrement marqué en période de crise, mais devient la norme dans les années $1840^{75}$.

68 AEN, MCE, CP 33/182, tr. 797, p. 340-341, arrêt du Conseil d'État du 28.2.1832; AEN, série Gendarmerie, AC 522/42, tr. 755, dossier 6/IX, projet d'arrêt Gendarmerie 28.2.1832.

69 Sur le rôle des huissiers (sautiers) et des justiciers (juges) et sur la justice criminelle, cf. Henry (1984, pp. 38-92); Courvoisier (1959, pp. 272-273). En 1844, il y en a encore 28 sautiers salariés par l'État (cf. AEN, AC 125, tr. 789, p. 56), sans compter ceux des communautés, employés et rétribués par ces dernières.

70 AEN, série Gendarmerie, AC 522/42, tr. 755, dossier 6/VIII, rapport directeur de la gendarmerie, 12.1.1829.

71 AEN, CP 34/21, tr. 775, p. 586-587; v. aussi arrêts déjà mentionnés des 28.2.1832 et 3.3.1832.

72 AEN, CP 34/21, f ${ }^{\circ}$ 588, 21.6.1831.

73 Cf. n. 34.

74 AEN, MCE, CP 33/186, tr. 797, p. 400: Service de la Gendarmerie pour les Comités de Défense, [Ponts-de-Martels] 5.3.1834 et Courvoisier (1982, p. 19).

75 En 1843, par exemple, l'augmentation d'effectif demandée à La Chaux-de-Fonds (on passe de quatre à six gendarmes) est justifiée par le fait de «pouvoir exercer une surveillance qui devient toujours plus active et nécessaire au dit lieu ». AEN, MCE, CP 33/205, tr. 785, p. 198, 9.8.1843. 


\section{6 - La création des postes d'inspecteurs de police par l'État (1845/1848)}

Un dernier cap est franchi le 24 février 1845, par la nomination de Jules François Vuithier, notaire et juge suppléant en la Cour de Justice de La Chaux-de-Fonds, au poste nouvellement créé d'inspecteur de police de La Chaux-de-Fonds, avant même que la ratification du roi pour la création de cet office ne soit intervenue ${ }^{76}$. Il est placé sous les ordres du chef de juridiction (agissant au nom du gouvernement), tandis que lui-même commande au poste de gendarmerie de la ville et que «c'est avec lui que la direction de police centrale correspondra pour tout ce qui tient au service de la gendarmerie» dans le but avoué «[d']assurer efficacement l'action de la police à La Chaux-de-Fonds ${ }^{77}$ ».

L'idée maîtresse est de soulager le maire de La Chaux-de-Fonds dans ses nombreuses occupations en établissant «un chef de police dont les fonctions embrasseraient tout ce qui ne serait pas directement judiciaire» (la gendarmerie neuchâteloise, avant 1848, n'est pas une police judiciaire). C'est donc la première fois qu'au niveau de l'État, les affaires de police sont détachées des fonctions de l'officier de juridiction et confiées à un fonctionnaire spécial, nouvellement créé à cet effet ${ }^{78}$ ! C'était une des demandes contenues dans le rapport d'A.-F. de Perregaux sur les améliorations de la police ${ }^{79}$.

Cette ultime création de Perregaux et l'augmentation du nombre de gendarmes demandée étant définitivement adoptée par l'administration neuchâteloise sur aval du roi ${ }^{80}$, le directeur de la police centrale peut démissionner en toute quiétude de son poste en $1845^{81}$. L'inspecteur ayant apparemment fait ses preuves, une charge identique est instaurée en toute hâte et sur le même pied au Locle en janvier 1848, dans l'espoir de contribuer ainsi à juguler la révolte qui sourd, mais il est trop tard pour éviter la chute du régime ${ }^{82}$.

\section{7 - La gendarmerie et le crépuscule du régime royaliste: essai de synthèse}

Le profil des successeurs d'A.-F. de Perregaux à la tête du corps de gendarmerie, Auguste Favre (1845-1846) et Charles Lardy $(1846-1848)^{83}$, tous deux des civils,

76 AEN, CP 34/23, tr. 775, p. 515, rubrique «Offices de l'État» et AEN, série Police centrale, AC 522/70, tr. 762, dossier 66/I, extrait du registre du Conseil d'État (MCE), 24.2.1845, nomination de l'inspecteur de police de La Chaux-de-Fonds.

77 AEN, MCE, CP 33/208, tr. 785, pp. 251-253, 10.2 .1845 et pp. 332-335, 20.2.1845.

78 La ville de Neuchâtel avait un peu d'avance en la matière, un inspecteur de police (aussi appelé inspecteur de la garde, de la police et des gendarmes) y officiant au moins dès 1842, cf. AN (1840-1848) et AEN, MCE, CP 33/202, tr. 785, pp. 295-296, 26.2.1842; p. 574, 6.4.1842; MCE, CP 33/213, tr. 785, p. $1004,6.11 .1847$.

79 Cf. supra.

80 AEN, MCE, CP 33/208, tr. 785, pp. 345-346: «Exercice de Police à La Chaux-de-Fonds » et arrêts du Conseil d'État, pp. 346-347, 24.2.1845.

81 AEN, MCE, CP 33/208, tr. 785, pp. 430-431, «congé de directeur de la police centrale. De Perregaux », arrêt du 10.3.1845.

82 AEN, MCE, CP 33/214, tr. 785, pp. 59-60, 12.1.1848; série Police centrale, AC 522/70, tr. 762, dossier 66/I, «Projet de règlement pour l'inspecteur de Police [...]», 19.1.1848 et MCE, CP 34/23, tr. 775, p. 523, arrêt réglementaire du 31.1.1848.

83 Augustin Favre (1805-1846), secrétaire personnel du maire de Pierre (1824), secrétaire de la commission de police centrale et de la gendarmerie (1824-1846). Directeur de la police centrale (mars 
dont aucun n'est plus Conseiller d'État, tend à démontrer que la mue de ce corps de l'État est provisoirement achevée en 1845. Sous la houlette de Perregaux, la gendarmerie est devenue, en l'espace de vingt ans, une branche à part entière de l'administration. L'institution a montré son utilité et fonctionne suffisamment bien pour qu'elle n'ait désormais plus besoin d'un membre du gouvernement à sa tête. La direction de police centrale ${ }^{84}$ est toujours rattachée au Département de justice et police, aux contours flous, dont elle ne dépend que vaguement. Mais elle n'a toujours aucun lien avec le Département militaire, qui gère les milices et les troupes de volontaires levées à l'occasion de désordres ou de menace pour l'ordre établi.

Pour la gendarmerie/police centrale, devenue un véritable outil du pouvoir, maintenir l'ordre intérieur est devenu une priorité. Les mesures policières mises en place en 1845 et en 1847-1848 attestent bien de la participation de plein fouet de la gendarmerie à la surveillance et à la tentative d'endiguement des nouvelles menées révolutionnaires. La création des deux postes d'inspecteurs au Locle et à La Chauxde-Fonds participe de cette dynamique. Les tâches confiées à la gendarmerie dans ce contexte troublé (mesures d'encadrement de la population, service journalier de nouvelles, collaboration avec les comités de défense, etc. $)^{85}$, relèvent bien de la police, et même de la police politique ${ }^{86}$. La facilité avec laquelle Lardy obtient l'attribution d'un nombre important de gendarmes surnuméraires pour renforcer sa police ( 24 sur 57 gendarmes en 1847, alors qu'en 1846 il n'y en avait que 37 en tout au service de l'État ${ }^{87}$ démontre que la gendarmerie est partie prenante aux mesures prises pour essayer de sauver le régime. Cette implication ne fait qu'attester de la réussite des transformations de la police opérées par A.-F. de Perregaux. D'une troupe vouée essentiellement à la répression de la mendicité, et sans renier cette dernière tâche, il a fait une force publique investie dans le maintien de l'ordre. Charles Lardy, dernier directeur de la police centrale royaliste, a vu sa fonction prendre encore plus d'ampleur, devenant un poste quasi à temps plein et prenant le pas sur toutes les autres charges qu'il occupe ${ }^{88}$. Une mutation professionnelle est en route.

Un autre élément saillant des changements opérés pendant ces années est la dichotomie entre la voie politique suivie par les autorités de la principauté, qui s'éloigne de plus en plus d'une certaine Suisse dont elles se méfient, et l'évolution d'une partie de l'administration, la police centrale, qui elle copie sans vergogne ses voisins suisses. En 1845 encore, le gouvernement neuchâtelois s'interroge sur la possibilité d'engager un nouveau gendarme. Il demande en conséquence à son directeur un état comparatif de la gendarmerie avec les gouvernements de Berne,

1845-août 1846), cf. Messager boiteux de Neuchâtel (1847, pp. 46-47); Christ (2009, p. 1251 n. 732). Sur Charles-Louis Lardy (1816-1875), avocat et docteur en droit, maire des Ponts, député, père du futur ministre de Suisse à Paris Charles-Édouard Lardy, cf. ibid., 1877, p. 34.

84 Synonyme de gendarmerie depuis 1832 .

85 Maeder (2005, pp. 384, 392-393).

86 AEN, série Événements 1831-1848, AC 522/36, tr. 752, Registre 19, dossiers I-VI: rapports du directeur de la police centrale, Lardy, au conseil d'État sur les affaires publiques, avril 1847-février 1848.

87 AEN, série Gendarmerie, AC 522/42, tr. 755, dossier 7/IV, rapport du directeur de la police centrale Lardy au Conseil d'État sur la répartition des 24 gendarmes surnuméraires, 12.1.1848.

88 AEN, Série Police centrale, AC 522/70, Tr. 762, dossier 66/I, brouillon de lettre, Lardy au Conseil d'État, 15/17.1.1848. 
Fribourg, Vaud et Genève ${ }^{89}$. La direction répond alors avec des chiffres concernant non seulement les quatre cantons précités, mais tous les cantons suisses (sauf Appenzell). Cet épisode atteste que la sphère helvétique constitue bien désormais l'étalon de Neuchâtel en matière de police.

\section{III - LA GENDARMERIE
ET LES DÉBUTS DE LA RÉPUBLIQUE (1848-1850)}

Le $1^{\text {er }}$ mars 1848 , la révolution renverse le régime monarchique en place et instaure la République. Pour ne pas répéter les erreurs de 1831, un gouvernement provisoire de la République et canton de Neuchâtel est immédiatement nommé et se met aussitôt au travail, notamment pour assurer le maintien de l'ordre et de la sécurité publique et éviter tout retour de flamme ${ }^{90}$.

\section{1 - Les mesures transitoires}

Au lendemain de la prise du pouvoir par les républicains, le gouvernement provisoire instaure des comités administratifs et de police provisoires dans les principales communes du pays. Autorisés à requérir la force armée ${ }^{91}$, ces comités sont chargés de représenter le gouvernement provisoire, exercent les fonctions de police, veillent au maintien de l'ordre, de la tranquillité publique, au respect des propriétés et à la sûreté des personnes, ainsi qu'à l'exécution des arrêtés du gouvernement provisoire.

Une fois le gouvernement légalisé par la première constitution neuchâteloise, entrée en vigueur le 3 mai 1848, et en l'absence d'une organisation militaire cantonale définitive, le Grand Conseil (parlement cantonal) décide d'organiser sans retard des corps armés qui prennent le relais des comités administratifs et de police, licenciés au début de l'été $1848^{92}$. Des gardes civiques sont ainsi instituées dans les principales localités du canton et partout où le Conseil d'État le juge nécessaire «pour le maintien de l'ordre et de la tranquillité publique ${ }^{93}{ }$. Elles sont placées sous la direction de ce dernier ou de ses agents directs (préfets et commissaires de police) et commandées par des officiers nommés par le gouvernement.

Pour autant, la gendarmerie ne disparaît pas. Le 14 mars 1848, le gouvernement provisoire décide son maintien provisoire sur le même pied qu'auparavant ${ }^{94}$. Peu de changements dans l'immédiat pour la police neuchâteloise, institution qui conserve

89 AEN, série Gendarmerie, AC 522/42, tr. 755, dossier 7/I, rapport de la direction de police centrale du 25 août 1845 .

90 Ce gouvernement existe du $1^{\mathrm{er}}$ mars au 3 mai 1848 avant de laisser place aux institutions élues. Sur les événements de 1848, cf. Barrelet (1993).

$91 \quad R L$ (I, pp. 8-9): arrêté du gouvernement provisoire ordonnant leur formation, 2.3.1848; formulaire portant leur nomination à la connaissance du public, 4.3.1848, $R L$ (I, p. 13); instructions à leur usage, 4.3.1848, RL (I, p. 14).

$92 B G C(1,1848$, p. 474), séance du 8.7.1848: lettres de remerciements aux commissaires du gouvernement et aux comités administratifs provisoires.

93 Loi et règlement pour les gardes civiques, 12.9.1848, $R L$ (I, pp. 382-383); règlement pour les gardes civiques, 10.10.1848, RL (I, pp. 384-387).

94 AEN, MCE, CP 33/215, tr. 785, p. 163, 14.3.1848. 
la confiance du gouvernement provisoire malgré le fait qu'elle soit l'œuvre des royalistes ${ }^{95}$.

Dans la foulée, pour parer au plus pressé, un commissaire provisoire de police est nommé à La Chaux-de-Fonds, pour contrôler les Montagnes ${ }^{96}$. Ce dernier porte d'abord l'ancien titre d'inspecteur lequel, bien que rapidement remplacé, atteste de la filiation de la charge, qui passe d'un régime à l'autre, mais révèle aussi la volonté rapide de rompre avec le passé en modifiant le titre de la fonction, renommée probablement à l'exemple de la France ${ }^{97}$. Le nouveau Conseil d'État poursuit ses réformes en nommant provisoirement, pour un an, trois commissaires de police ${ }^{98}$, en remplacement des trois inspecteurs qui avaient été mis en place par les royalistes. En 1848 et 1849 , trois commissaires de police officient donc en concomitance avec la gendarmerie: un militaire de métier à Neuchâtel (Charles-Jules Mathey, capitaine) et deux civils, Auguste Jeanneret-Grosjean, au Locle, et Pierre-Louis Évard-Bourquin, à La Chaux-de-Fonds, confirmé dans son poste ${ }^{99}$. Ces nominations démontrent que les autorités hésitent alors encore entre une police civile, incarnée par le commissaire de police (vraisemblablement à l'exemple des municipalités françaises ${ }^{100}$ ) et une gendarmerie, dont le statut n'a pas encore été fixé. La seconde option l'emporte rapidement, puisque les postes de commissaires sont supprimés fin $1849^{101}$, leurs attributions policières passant aux préfets nouvellement créés.

Quant à la police centrale, la république en fait une administration provisoire en 1850 , la Direction de la police centrale, dont relève la gendarmerie (après avoir été soumise au département militaire $)^{102}$. Le lien établi entre les deux administrations sous le précédent régime est donc maintenu.

\section{2 - La gendarmerie républicaine}

La question du statut civil ou militaire de la gendarmerie est résolue en décembre 1849. L'article $1^{\text {er }}$ de la loi sur la gendarmerie stipule en effet que la gendarmerie est dès lors un corps militaire placé sous la direction du Département militaire ${ }^{103}$. Le Grand Conseil a tranché là où les royalistes n'ont jamais osé franchir le pas. C'est une première pour Neuchâtel, qui n'a jusque-là pas connu de police à statut militaire. Si la transformation de la gendarmerie en corps militaire ne pose pas de problème, la nouveauté de cette organisation est néanmoins soulevée lors de la discussion de la loi au parlement cantonal, notamment par Charles Lardy, ancien chef de la police centrale et député. Ce dernier voit d'un mauvais œil le nouveau statut du

95 Ce qui n'est pas le cas à Fribourg, par exemple, qui la supprime lors de la Révolution radicale de 1846. Foerster, Python (1979).

96 AEN, MCE, CP 33/215, tr. 785, p. 252: nomination d'un commissaire provisoire de police (Évard Bourquin) à La Chaux-de-Fonds, mars 1848 .

97 Sur le commissaire de police, cf. Kalifa, Karila-Cohen (2008).

$98 B G C$, vol. 1, 1848, p. 474, séance du 8.7.1848 et $B G C$ (vol. 2, 1849, pp. 241-243), traitement des commissaires police, 2.11.1848.

$99 \quad A O(1849$, p. 76), rubrique Principaux agents du Conseil d'État.

100 Berlière (1996, pp. 22-29).

101 Ils n'apparaissent plus au budget de l'État, cf. $B G C(4,1849)$ et $B G C(5,1850$, p. 404).

102 AO (1850).

103 Loi sur la gendarmerie, 21.12.1849], $R L$ (II, pp. 245-250), exécutoire dès le $1^{\text {er }}$ janvier 1850. 
gendarme, jugé moins stable que le précédent. Lardy explique en effet «[qu']on cherchait à avoir pour gendarmes des hommes mariés, calmes, rangés, tandis que les gendarmes étant soldats, il sera difficile d'avoir des hommes mariés, et l'on sera exposé à plus de taquineries et d'abus de pouvoir de la part de jeunes gens non mariés ${ }^{104}$.

Quoiqu'il en soit du bien-fondé de cette affirmation, cette troupe, composée de 79 hommes, est désormais commandée par un capitaine, tous les grades se déclinant sur le modèle militaire suisse, ce qui a pour conséquence la disparition du terme de brigadier. L'ancienne répartition territoriale est abandonnée, la gendarmerie adoptant une organisation fondée sur les nouveaux districts. Tout, dans la nouvelle loi, concourt à souligner l'organisation militaire du corps. Quant aux anciens gendarmes, le Grand-Conseil est chargé de statuer sur le sort, qu'ils soient ou non réengagés dans le nouveau corps.

La gendarmerie, dotée d'un nouveau règlement ${ }^{105}$, est instituée pour veiller à la sûreté publique, maintenir l'ordre et assurer l'exécution des lois. Son cahier des charges est remis à jour, les tâches clarifiées et son service décrit avec soin. Les hommes du corps sont à l'avenir soumis aux lois militaires. Quant à l'institution, elle est placée sous la double direction du Département militaire, pour tout ce qui concerne son organisation, et du Département de police, en ce qui concerne notamment la police administrative et judiciaire, l'exécution des mandements de justice et le maintien de l'ordre public.

L'organisation de la gendarmerie et les départements dont elle dépend pourraient laisser entendre qu'ils sont calqués sur la police militaire française ${ }^{106}$. Or, sous le régime royaliste, cette filiation est au mieux indirecte. Que l'institution française ait, en 1850, directement inspiré les Neuchâtelois ou que l'emprunt se soit fait via les polices suisses n'a donc pas beaucoup d'importance. Car finalement, il aura fallu attendre la République, 40 ans après la fondation de la gendarmerie, pour que le modèle gendarmique français s'impose.

Le Conseil d'Etat entérine définitivement la voie d'une gendarmerie organisée en corps militaire en nommant son premier chef en la personne du Bernois CharlesFrédéric Flühmann (1814-1881), capitaine et commandant de la gendarmerie de 1850 à 1867, personnage à la riche expérience militaire ${ }^{107}$. À partir de 1851 , la gendarmerie fait donc tout naturellement son apparition dans l'Annuaire officiel de la République et canton de Neuchâtel ${ }^{108}$, au nombre du personnel militaire cantonal.

\section{NEUCHÂTEL, UN MODĖLE ORIGINAL DE POLICE?}

Bien qu'elle en prenne le nom en 1809, la gendarmerie neuchâteloise est longtemps demeurée bien plus proche de la maréchaussée, dont elle est issue, que du

$104 B G C(5,1849$, pp. 324-333, spéc. pp. 330-332), séance du 17.12 .1849 et pp. 400-413, séance du 21.12.1849, part. p. 405.

105 Règlement pour le corps de gendarmerie, 22.3.1850, RL (III, pp. 125-157).

106 Sur la gendarmerie française, cf. Luc (2002, 2005); Berlière (1996, pp. 16-19).

107 Depuis 1838, il fait en effet partie des cadres militaires bernois et prend une part active à la révolution de 1848, en dirigeant les troupes républicaines de La Chaux-de-Fonds, puis comme commandant de place au Locle pour le gouvernement provisoire. $B S$, rubrique «Militär-Etat des Kantons Bern», 1838-1849; AO (1850-1867); Messager boiteux de Neuchâtel, 1917, pp. 73-76. 
corps militaire français éponyme. Sa création elle-même est due non à l'intervention d'un expert français, mais à la volonté du gouvernement neuchâtelois lui-même. Malgré le changement de nom, l'organisation et les tâches varient peu d'une institution à l'autre. Mais plus encore, la permanence de la structure d'Ancien régime se lit dans les attributions de justice prévôtale que la gendarmerie de la principauté prussienne et canton suisse conserve et exerce jusqu'en 1848. Contrairement à ce qui se passe en Europe au début du XIX ${ }^{\mathrm{e}}$ siècle, à Neuchâtel, si l'on adopte bien le nom de «gendarmerie» pour le corps de police, l'ancienne structure persiste, alors qu'ailleurs, dans presque tous les territoires conquis par la France de Napoléon, le modèle gendarmique s'impose, bien que parfois sous un nom différent. Le cas de Neuchâtel, qui prend le contrepied de l'Europe, constitue donc bien une spécificité à cet égard. C'est un parfait contre-exemple au modèle gendarmique français, duquel elle diffère en de nombreux points, du moins à ses débuts: force publique civile et non militaire, dénuée de commandement militaire, pas d'organisation en brigades typique du maillage territorial français, pas d'encasernement des troupes, etc. En fait, le corps neuchâtelois n'a aucun lien organique avec la gendarmerie française, dont elle ne fait que reprendre le nom!

La conversion, d'abord timide, ne débute que dans les années 1820 , suite à la nomination d'A.-F. de Perregaux au poste de directeur de la gendarmerie. Cette dernière se mue alors peu à peu en un organisme beaucoup plus proche d'une gendarmerie militarisée «à la française». L'influence du modèle gendarmique français sur l'organisation neuchâteloise n'est cependant qu'indirecte et emprunte des voies détournées. Ainsi, la gendarmerie royaliste s'est construite au carrefour des influences française, prussienne et suisse, qui ne l'ont pas toutes façonnée avec la même force. L'imitation des autres polices suisses devient en effet la source d'inspiration prééminente au fil des ans. Du « refus victorieux du modèle français ${ }^{109}$ qui prévaut au début du XIX ${ }^{\mathrm{e}}$ siècle, l'on glisse lentement vers son intégration, qui s'opère par les biais décrits supra. Par petites touches, au gré d'adaptations successives, une institution véritablement au service du pouvoir voit le jour, institution dont le développement coöncide avec celui de l'administration de l'État, particulièrement après 1831. Transformée en une gendarmerie à l'efficacité accrue sous la houlette de Perregaux, l'on ne saurait taire l'apport de l'étude des pratiques policières. Leur examen révèle le glissement de la gendarmerie, fusionnée avec la commission de police centrale, vers des tâches de surveillance de la population, emploi dont les rares règlements ne font pas mention.

Mais il ne faut pas perdre de vue non plus que quel que soit le succès des réformes de Perregaux, la gendarmerie était insuffisante pour maintenir seule l'ordre, tâche qui n'était pas paradoxalement sa vocation première. Le recours aux troupes militaires et aux volontaires demeurait nécessaire, ce que les événements de 1831 et 1848 ont montré. En l'état de la recherche, l'on ne peut cependant affirmer clairement à quel degré la gendarmerie neuchâteloise a fait fonction de vecteur de pénétration de l'idée de l'Etat-nation dans le pays ${ }^{110}$, puisque cette police est avant tout une émanation du gouvernement oligarchique neuchâtelois, de plus en plus

109 Tournure empruntée à Henry (2003), qui fait la démonstration de la résistance de l'administration de la principauté à l'acculturation par les modèles français.

110 Ce rôle important de la gendarmerie a notamment été démontré par les travaux de Emsley et Broers, cf. supra. 
contesté. Peut-être a-t-elle contribué à forger une identité républicaine a contrario, si l'on peut dire? Cela doit encore être démontré.

Le statut civil des gendarmes représente la deuxième spécificité neuchâteloise de taille. Cette particularité verrouille pour longtemps la réorganisation du corps de la gendarmerie selon le modèle militaire français, adopté de longue date partout ailleurs en Suisse et auquel les chefs neuchâtelois aspirent sans jamais oser franchir le pas. Cet état de fait résulte, pour partie de choix conscients, mais également des blocages inhérents au système politico-administratif de l'Ancien régime neuchâtelois ${ }^{11}$. Sans doute que l'étude du recrutement des gendarmes permettrait de nuancer ou au contraire d'affirmer le poids de l'option «paramilitaire». Mais cet examen, s'il avait été possible, aurait largement dépassé le cadre de ce travail ${ }^{112}$, qui a donc sciemment privilégié une approche dans le sens d'une «histoire politique de l'État ${ }^{113}$ aux fins de la démonstration.

Lors du passage à la République, les nouvelles autorités ne font pas table rase du passé en balayant du revers de la main toutes les créations du régime honni. Au contraire, elles en reprennent quasiment l'intégralité, en matière de police s'entend. Nous l'avons vu, les éphémères commissaires sont les descendants directs des inspecteurs créés par les royalistes. Le nouveau gouvernement garde de l'ancien régime, même temporairement, ce qu'il juge momentanément utile. C'est exactement ce qui se passe dans le cas de l'institution gendarmique. L'avènement de la république radicale en 1848 ne remet pas son existence en cause, bien qu'elle soit l'héritière directe de la gendarmerie royaliste. En conservant l'institution, le nouveau régime lui témoigne sa confiance, considérant qu'elle a servi l'État et non les affidés de la Prusse. Ainsi, c'est la république qui achève la mutation définitive de la gendarmerie en un corps militaire, mettant un terme aux louvoiements antérieurs. L'option d'une police à statut civil est alors enterrée pour de longues années. La justice prévôtale est définitivement supprimée, les tâches de la gendarmerie sont clarifiées et elle devient officiellement une police judiciaire.

Peut-être, enfin, pourrait-on voir dans le succès du modèle militaire l'influence du rôle unificateur et pacificateur de l'armée suisse des radicaux, victorieuse de la guerre civile dont est issue la Confédération de $1848^{114}$.

Philippe Hebeisen
Institut d'histoire, Université de Neuchâtel
Espace Louis-Agassiz 1
$\mathrm{CH}-2000$ Neuchâtel
philippe.hebeisen@unine.ch

111 Sur cette question, cf. Henry (1999b).

112 Du fait de l'organisation civile de la gendarmerie, couplée au hasard archivistique, les AEN n'ont conservé aucun dossier personnel concernant les gendarmes, pour autant que ceux-ci aient bien sûr existé. Pour un bel exemple d'étude de cas à partir de tels dossiers, cf. Houte (2000).

113 Laurent (2005, p. 768).

114 Issu d'une présentation faite lors des $4^{\text {e }}$ Journées CIRSAP (Lille-Villeneuve d'Ascq, 4-6 décembre 2008), consacrées à la Construction et circulation des savoirs policiers en Europe centrale et septentrionale, $X V I I I^{e}-X I X^{e}$ siècles, cet article a profité des remarques de certains intervenants au colloque et de mes deux relecteurs anonymes, ainsi que des conseils de Serge Reubi; qu'ils soient ici tous remerciés. 


\section{SOURCES IMPRIMÉES}

Almanach de Neuchâtel en Suisse pour l'an de grâce... [cité AN], 1808-1848.

Annuaire officiel de la République et canton de Neuchâtel pour... [cité AO], [S.l.]: [s.n.], 1849 sqq.

Bernischer Staatskalender auf das Jahr...= Annuaire officiel du canton de Berne [cité BS], diverses années.

Bulletin officiel des séances du Corps législatif de la Principauté et canton de Neuchâtel [cité BOCL], [S.1.], [s.n.], 1831-1847.

Bulletin officiel des délibérations du Grand Conseil de la République et canton de Neuchâtel [cité BGC], [Neuchâtel], [Chancellerie d'État], 1848-1915.

Recueil de pièces officielles concernant la Principauté de Neuchâtel et Valangin [cité RPO], Neuchâtel, [Principauté de Neuchâtel et Valangin], 1827-1849.

Recueil des lois, décrets, et autres actes du gouvernement de la République et canton de Neuchâtel [cité RL], [S.1.], [s.n.], 1849-1886.

\section{BIBLIOGRAPHIE}

Arlettaz, S., Regards sur les institutions et les droits fondamentaux de la République indépendante du Valais (1802-1810), in Dufour, A., Hanisch, T., Monnier, V. (dir.), Bonaparte, la Suisse et l'Europe: actes du Colloque européen d'histoire constitutionnelle pour le bicentenaire de l'Acte de Médiation (1803-2003), Bruxelles [etc.], Bruylant [etc.], 2003, pp. 257-275.

Arnet, H., 150 Jahre Kantonspolizei Bern, 1804-1954, Bern, Kümmerly \& Frey, 1954.

Bachelin, A., Gendarmes neuchâtelois (1840), Musée Neuchâtelois, 1890, pp. 76-77.

Bandelier, A., Porrentruy, sous-préfecture du Haut-Rhin : un arrondissement communal sous le Consulat et l'Empire, 1800-1814: l'Évêché de Bâle et le pays de Montbéliard à l'époque napoléonienne, Neuchâtel, La Baconnière, 1980.

Barrelet, J.-M. (dir.), Histoire du Pays de Neuchâtel, vol. 3, Hauterive, Gilles Attinger, 1993.

Bauer, E., Vie militaire, Neuchâtel, Comité directeur du centenaire de la République neuchâteloise, 1948.

Berlière, J.-M., Le monde des polices en France: XIX $-X X^{e}$ siècles, Bruxelles, Complexe, 1996.

Bieri, R., Die Landjägerkorps, in Jung, J. (ed.), Der Bockenkrieg 1804 : Aspekte eines Volkaufstandes, Zurich, Verlag NZZ, 2004, pp. 131-167.

Broers, M., La gendarmerie et le maintien de l'ordre public dans l'Italie napoléonienne, 18001814: institutions françaises et société baroque; la culture et la police, in Rousseaux, X., Dupont-Bouchat, M.-S., Vael, C. (dir.), Révolutions et justice pénale en Europe, modèles français et traditions nationales (1780-1830)/Revolution and criminal justice, french models and national traditions (1780-1830), Paris, L'Harmattan, 1999, pp. 179-187.

Brouillet, P. (dir.), De la Maréchaussée à la Gendarmerie : histoire et patrimoine, MaisonsAlfort, Service historique de la Gendarmerie nationale, 2003.

Christ, T., Riard, S., Du réduit communal à l'espace national: le statut des étrangers dans le canton de Neuchâtel 1750-1914, Hauterive, Gilles Attinger; Neuchâtel, Société d'histoire et d'archéologie de Neuchâtel, 2000.

Christ, T., Des solidarités coutumières à la bienfaisance privée: l'État et les pauvres à Neuchâtel (1773-1830), Neuchâtel, Université de Neuchâtel Faculté des lettres et sciences humaines, 2009, 3 vol., Thèse de doctorat, url [http://doc.rero.ch/record/12517 ?ln =fr]. 
Courvoisier, J., Le maréchal Berthier et sa Principauté de Neuchâtel : 1806-1814, Neuchâtel, Société d'histoire et d'archéologie, 1959.

Courvoisier, J., Les gardes urbaines et les conseils de défense de l'Ancien Régime, 18311848, Musée Neuchâtelois, 1982, pp. 4-34.

Courvoisier, J., Parenthèse ou transition? Le règne d'Alexandre Berthier (1806-1814), Revue historique neuchâteloise, 2002, 3-4, pp. 215-228.

Dictionnaire historique de la Suisse, Hauterive, G. Attinger, 2002- [cité $D H S, \mathrm{n}^{\circ}$ vol.]; version électronique sous [www.dhs.ch].

Emsley, C., A typology of nineteenth-century police, Crime, Histoire et Sociétés/Crime, History and Societies, 1999a, 3, 1, pp. 29-44.

Emsley, C., Gendarmes and the state in nineteenth-century Europe, Oxford, Oxford University Press, 1999b.

Foerster, H., Python, C., 1804-1979: 175 ans gendarmerie fribourgeoise/175 Jahre Freiburger Polizei, Fribourg, Gendarmerie cantonale, 1979.

Gugger, R., Verwaltung auf Distanz: Preussen und Neuenburg 1831-1848, in Pfister, U., Tribolet, M. de (Eds), De l'État féodal à l'État gestionnaire. Naissance et développement de l'administration moderne (Itinera, 21), Bâle, Schwabe \& Co., 1999, pp. 105-115.

Gugger, R., La légalité comme boussole. Neuchâtel à la recherche de sa place en Suisse (1831-1848), in Henry, P., Barrelet, J.-M. (dir.), Sujets ou Citoyens? Neuchâtel avant la Révolution de 1848, Neuchâtel, Université de Neuchâtel, Genève, Droz, 2005, pp. 19-97.

Henry, P., Crime, justice et société dans la Principauté de Neuchâtel au XVIII ${ }^{e}$ siècle (17071806), Neuchâtel, La Baconnière, 1984.

Henry, P., Jelmini, J.-P. (dir.), Histoire du Pays de Neuchâtel, vol. 2, Hauterive, Gilles Attinger, 1991.

Henry, P., Les débuts de la statistique criminelle et la naissance de la prison à Neuchâtel. Charles François de Marval 1801-1880, in Henry, P., Tribolet, M. de, (dir.), In dubiis libertas. Mélanges d'histoire offerts au professeur Rémy Scheurer, Hauterive, Gilles Attinger, 1999a, pp. 313-335.

Henry, P., Entre archaïsme et modernité: l'évolution du droit pénal neuchâtelois de 1814 à 1848, in Henry, P. (dir.), Conservatisme, réformisme et contestation: aux origines de la Révolution neuchâteloise de 1848, Neuchâtel, Université de Neuchâtel, 1999b, p. 87-100.

Henry, P., La principauté de Neuchâtel de 1803 à 1813, ou le refus victorieux des modèles français, in Dufour, A., Hanisch, T., Monnier, V. (Dir.), Bonaparte, la Suisse et l'Europe: actes du Colloque européen d'histoire constitutionnelle pour le bicentenaire de l'Acte de Médiation (1803-2003), Bruxelles [etc.], Bruylant [etc.], 2003, pp. 277-298.

Henry P., Neuchâtel français?: le rattachement de la principauté à l'Empire napoléonien (1806-1814), in Mottu-Weber, L., Droux, J. (Eds), Genève française 1798-1813: nouvelles approches: actes du colloque tenu du 12 au 14 novembre 1998, Genève, Société d'histoire et d'archéologie de Genève, 2004, pp. 75-93.

Houte, A.-D., Gendarmes et gendarmerie dans le département du Nord (1814-1852), Maisons-Alfort, SHGN et Phénix Éditions, 2000.

Kalifa, D., Karila-Cohen, P. (dir.), Le commissaire de police au XIX siècle, Paris, Publications de la Sorbonne, 2008.

Laurent, S., Aux origines de la «guerre des polices »: militaires et policiers du renseignement dans la République (1870-1914), Revue historique, 2005, CCCXIV, 4, pp. 767-791.

Lepetit, G., Les hommes de la gendarmerie d'Espagne (1809-1814), Force Publique. Revue de la Société Nationale Histoire et Patrimoine de la Gendarmerie, Les gendarmes, la gendarmerie et la guerre. Actes du colloque du 21 octobre 2005, 2006, 1, pp. 33-47. 
Lepetit, G., «Des enfants de la Grande Famille»? Les compagnies de gendarmerie autochtones dans le nord de l'Espagne (1810-1813), Revue historique des armées, 2009, 255 , pp. 50-60.

Lévy, N., Modalités et enjeux de la circulation des savoirs policiers: un modèle français pour la police ottomane?, Revue d'histoire des sciences humaines, 2008, 19, 2, pp. 11-27.

Luc, J.-N., (dir.), Gendarmerie, état et société au XIX siècle, Paris, Publications de la Sorbonne, 2002.

Luc, J.-N. (dir.), Histoire de la maréchaussée et de la gendarmerie. Guide de recherche, Maisons-Alfort, SHGN, 2005.

Lüdtke, A., «Gemeinwohl», Polizei und «Festungspraxis»: staatliche Gewaltsamkeit und innere Verwaltung in Preussen, 1815-1850, Göttingen, Vandenhoeck und Ruprecht, 1982.

Maeder, A., En marche vers la Révolution: sédition et répression entre 1831 et 1848, in Henry, P., Barrelet, J.-M. (dir.), Sujets ou Citoyens? Neuchâtel avant la Révolution de 1848, Neuchâtel, Université de Neuchâtel, Genève, Droz, 2005, pp. 381-396.

Milliot, V., Mais que font les historiens de la police?, in Berlière, J.-M., Denys, C., Kalifa, D., Milliot, V., Métiers de police. Être policier en Europe, XVIII ${ }^{-}$XX $X^{e}$ siècles, Rennes, PUR, 2008, pp. 9-34.

Montandon, L., Faessler, F., Schnegg, A., Roulet, L.-E., Neuchâtel et la Suisse, Neuchâtel, Chancellerie d'État, P. Attinger, 1969.

Perregaux, C., La chasse aux gueux au XVIII ${ }^{\mathrm{e}}$ siècle, Musée neuchâtelois, 1900, pp. 101-109, 157-168; 1901, pp. 104-106.

Porret, M., Administration, police, censure et esprit publique à Genève pendant la période française, in Dufour, A., Hanisch, T., Monnier, V. (dir.), Bonaparte, la Suisse et l'Europe : actes du Colloque européen d'histoire constitutionnelle pour le bicentenaire de l'Acte de Médiation (1803-2003), Bruxelles [etc.], Bruylant [etc.], 2003, pp. 299-320.

Rousseaux, X., Entre politique, économie, société et culture: réflexions historiographiques sur deux siècles d'histoire du crime et de la criminalisation, in Opitz, C., Studer, B., Tanner, J. (Eds), Criminaliser, décriminaliser, normaliser, Zurich, Chronos, 2006, pp. 17-54.

Scheurer, R., Roulet, L.-E., Courvoisier, J., Histoire du Conseil d'État neuchâtelois, des origines à 1945, Neuchâtel, Chancellerie d'État, 1987.

Suratteau, J.-R., Le département du Mont-Terrible sous le régime du Directoire: (17951800): étude des contacts humains, économiques et sociaux dans un pays annexé et frontalier, Paris, Les Belles Lettres, 1965. 